\title{
Beyond Wiener-Askey Expansions: Handling Arbitrary PDFs
}

\author{
Xiaoliang Wan ${ }^{1}$ and George Em Karniadakis ${ }^{1}$
}

Received October 12, 2004; accepted (in revised form) August 2, 2005; Published online December 23, 2005

\begin{abstract}
In this paper we present a Multi-Element generalized Polynomial Chaos (MEgPC) method to deal with stochastic inputs with arbitrary probability measures. Based on the decomposition of the random space of the stochastic inputs, we construct numerically a set of orthogonal polynomials with respect to a conditional probability density function (PDF) in each element and subsequently implement generalized Polynomial Chaos (gPC) locally. Numerical examples show that ME-gPC exhibits both $p$ - and $h$-convergence for arbitrary probability measures.
\end{abstract}

KEY WORDS: Uncertainty; polynomial chaos; stochastic differential equation.

\section{INTRODUCTION}

Due to the rapid developments in algorithm and computational capability, accurate uncertainty modeling by non-statistical approach has received great attention in recent years. An effective approach based on the the homogeneous chaos theory of Wiener [1] is polynomial chaos, which has been successfully used in mechanics [2,3]. The original form of polynomial chaos is a spectral expansion based on the Hermite orthogonal polynomials in terms of Gaussian random variables. This approach was extended into a broader framework, called "generalized Polynomial Chaos (gPC)", in [4], which employs a broad family of classical orthogonal polynomials (the Askey scheme) as the expansion basis to represent nonGaussian process more efficiently. Within the framework of Wiener-chaos, a Wiener-Haar method based on wavelets was recently studied in $[5,6]$.

\footnotetext{
${ }^{1}$ Division of Applied Mathematics, Brown University, Providence, RI 02912, USA. E-mail: xlwan@dam.brown.edu,gk@dam.brown.edu
} 
Based on the finite element framework, polynomial chaos was studied theoretically in [7]; in particular, this work focused on the $h$-type discretization with $h$ being the size of elements in random space. More theoretical developments along the same lines are reported in [8-10], where the stochastic finite element (SFE) is proposed.

Despite the success on some applications, polynomial chaos expansions can be inefficient or convergence may even fail for problems requiring long-term integration or problems involving discontinuities induced by random inputs $[5,11]$, since increasing the order of polynomial chaos can give rise to a undesirable complicated system of differential equations or does not help at all. The SFE method reduces the stochastic problem to a high-dimensional deterministic one, and subsequently employs deterministic finite element solvers, which rely on $h$-type discretization for enhanced robustness as well as high accuracy. In [11], a ME-gPC method for uniform distribution was presented, which can achieve $h p$-convergence with $p$ being the polynomial order of gPC. The ME-gPC method is based on a decomposition of the random space of stochastic inputs, and maintains an orthogonal polynomial basis in each random element. For uniform random variables, such orthogonality can be inherited naturally due to the nice properties of uniform distribution. However, for an arbitrary probability measure, the orthogonality will be ruined by the random decomposition. In this paper, we generalize the ME-gPC method for an arbitrary probability measure, where the orthogonality of the polynomials in each random element can be maintained by a fast numerical reconstruction. The result is a new ME-gPC method that can achieve $h p$-convergence efficiently for any probability measure.

\section{ME-gPC METHOD}

In this section we present the multi-element generalized polynomial chaos (ME-gPC) method for random variables with arbitrary probability measures.

Let $(\Omega, \mathcal{F}, P)$ be a probability space and

$$
\boldsymbol{X}:(\Omega, \mathcal{F}) \rightarrow\left(\mathbb{R}^{d}, \mathcal{B}^{d}\right)
$$

be a $\mathbb{R}^{d}$-valued continuous random variable. Let $f(\boldsymbol{x})$ be the PDF of $\boldsymbol{X}$ defined on $B=\times_{i=1}^{d}\left[a_{i}, b_{i}\right]$, where $a_{i}$ and $b_{i}$ are finite or infinite in $\mathbb{R}$. We define a decomposition $\mathbf{D}$ of $B$ in the following way

$$
\mathbf{D}=\left\{\begin{array}{l}
B_{k}=\left[a_{k, 1}, b_{k, 1}\right) \times\left[a_{k, 2}, b_{k, 2}\right) \times \cdots \times\left[a_{k, d}, b_{k, d}\right], \\
B=\bigcup_{k=1}^{N} B_{k}, \\
B_{k_{1}} \bigcap B_{k_{2}}=\emptyset, \quad \text { if } k_{1} \neq k_{2},
\end{array}\right.
$$


where $k, k_{1}, k_{2}=1,2, \ldots, N$. Based on the decomposition $\mathbf{D}$, we define the following indicator random variables

$$
I_{B_{k}}= \begin{cases}1 & \text { if } \boldsymbol{X} \in B_{k} \\ 0 & \text { otherwise }\end{cases}
$$

It is obvious that

$$
\operatorname{Pr}\left(I_{B_{k}}=1\right)=\int_{B_{k}} f(\boldsymbol{x}) \mathrm{d} \boldsymbol{x} .
$$

Given any point $\boldsymbol{q}=\left(q_{1}, q_{2}, \ldots, q_{d}\right)$, we use $\boldsymbol{X} \leqslant \boldsymbol{q}$ to denote $x_{i} \leqslant q_{i}$ for $i=1,2, \ldots, d$. According to the properties of probability measure, we can obtain

$$
\operatorname{Pr}(\boldsymbol{X} \leqslant \boldsymbol{q})=\sum_{k=1}^{N} \operatorname{Pr}\left(\boldsymbol{X} \leqslant \boldsymbol{q} \mid I_{B_{k}}=1\right) \operatorname{Pr}\left(I_{B_{k}}=1\right) .
$$

Eq. (2.5) implies that we can define a new random variable $\boldsymbol{X}_{k}$ subject to a conditional PDF

$$
\hat{f_{k}}\left(\boldsymbol{x}_{k} \mid I_{B_{k}}=1\right)=\frac{f\left(\boldsymbol{x}_{k}\right)}{\operatorname{Pr}\left(I_{B_{k}}=1\right)}
$$

in each random element $B_{k}$. Instead of considering $\boldsymbol{X}$ in the whole random space, we first approximate the random field locally within each random element $B_{k}$ by $\mathrm{gPC}$ with respect to $\boldsymbol{X}_{k}$, then reconstruct the original random field. Once we get the localized random field, we can compute any statistics in the following way

$$
\int_{B} g(u(\boldsymbol{x})) f(\boldsymbol{x}) \mathrm{d} \boldsymbol{x} \approx \sum_{k=1}^{N} \operatorname{Pr}\left(I_{B_{k}}=1\right) \int_{B_{k}} g\left(\hat{u}_{k}\left(\boldsymbol{x}_{k}\right)\right) \hat{f}\left(\boldsymbol{x}_{k} \mid I_{B_{k}}=1\right) \mathrm{d} \boldsymbol{x}_{k},
$$

where $g(\cdot) \in L_{1}(\Omega, \mathcal{F}, P)$ is any function of random field $u(\boldsymbol{X})$, and $\hat{u}_{k}\left(\boldsymbol{X}_{k}\right)$ denotes the approximated local random field in element $B_{k}$ by $\mathrm{gPC}$.

Let $u_{e}\left(\boldsymbol{X}_{k}\right)$ denote the exact form of local random field determined by a stochastic differential equation. We know that $u(\boldsymbol{X})$ in element $B_{k}$ should have the same form with $u_{e}\left(\boldsymbol{X}_{k}\right)$ because $\boldsymbol{X}$ and $\boldsymbol{X}_{k}$ have the same range $\times_{i=1}^{d}\left[a_{k, i}, b_{k, i}\right)$ in element $B_{k}$ and their forms only depend on a particular stochastic differential euqation. We know that $\hat{u}_{k}\left(\boldsymbol{X}_{k}\right)$ in polynomial chaos expansion converges to $u_{e}\left(\boldsymbol{X}_{k}\right)$ in the $L_{2}$ sense with respect to 
$\hat{f}_{k}\left(\boldsymbol{x}_{k} \mid I_{B_{k}}=1\right)[12,4,9]$. Since $\hat{f}_{k}\left(\boldsymbol{x}_{k} \mid I_{B_{k}}=1\right)$ is the same with the density function $f(\boldsymbol{x})$ except a constant factor, we can obtain that

$$
\begin{aligned}
& \sum_{k=1}^{N} \operatorname{Pr}\left(I_{B_{k}}=1\right) \int_{B_{k}} g\left(\hat{u}_{k}\left(\boldsymbol{x}_{k}\right)\right) \hat{f}\left(\boldsymbol{x}_{k} \mid I_{B_{k}}=1\right) \mathrm{d} \boldsymbol{x}_{k} \\
& \quad=\sum_{k=1}^{N} \int_{B_{k}} g\left(\hat{u}_{k}\left(\boldsymbol{x}_{k}\right)\right) f\left(\boldsymbol{x}_{k}\right) \mathrm{d} \boldsymbol{x}_{k} \\
& \quad \rightarrow \sum_{k=1}^{N} \int_{B_{k}} g\left(u_{e}\left(\boldsymbol{x}_{k}\right)\right) f\left(\boldsymbol{x}_{k}\right) \mathrm{d} \boldsymbol{x}=\int_{B} g(u(\boldsymbol{x})) f(\boldsymbol{x}) \mathrm{d} \boldsymbol{x},
\end{aligned}
$$

where the arrow indicates a $L_{2}$ convergence on $(\Omega, \mathcal{F}, P)$.

To this end, we give a procedure of random decomposition. Based on such a random decomposition, we have the following choices to solve the stochastic differential equations:

1. The basis of finite element method can be introduced, which yields a variation of the stochastic finite element (SFE) method [7-9].

2. Based on the conditional PDF in each element, we can construct a new set of orthogonal polynomials in terms of $\boldsymbol{X}_{k}$ and implement a truly generalized polynomial chaos (gPC) $[2,4,10]$ element by element.

In this work, we focus on the second choice. It is a distinctive feature of orthogonal polynomials, compared to other orthogonal systems, that they satisfy a three-term recurrence relation,

$$
\begin{gathered}
\pi_{k, i+1}(t)=\left(t-\alpha_{k, i}\right) \pi_{k, i}(t)-\beta_{k, i} \pi_{k, i-1}(t), \quad i=0,1, \ldots, \\
\pi_{k, 0}(t)=1, \quad \pi_{k,-1}(t)=0,
\end{gathered}
$$

where coefficients $\alpha_{k, i}$ and $\beta_{k, i}$ are uniquely determined by the weight function, and $\left\{\pi_{k, i}(t)\right\}$ is a set of (monic) orthogonal polynomials in element $k$,

$$
\pi_{k, i}(t)=t^{i}+\text { lower-degree terms, } \quad i=0,1, \ldots
$$

Given a weight function we can first compute the recurrence coefficients $\alpha_{k, i}$ and $\beta_{k, i}$ numerically, then determine the Gauss-type quadrature points and corresponding integration weights based on $\alpha_{k, i}$ and $\beta_{k, i}$. In this work, we use the Stieltjes procedure and the Lanczos algorithm $[13,14]$ to compute the recurrence coefficients $\alpha_{k, i}$ and $\beta_{k, i}$. For efficiency, we prefer to use relative low-order polynomials, e.g. $p=3$ to 5 , in the ME-gPC 
method, which means that we can construct the new orthogonal polynomials on-the-fly in the pre-processing stage. For example, the Beta distribution $\mathrm{B} e(1,2)$ on $[-1,1]$ can be decomposed into 1000 equidistant random elements in about 0.1 seconds on an AMD $1.5 \mathrm{GHz} \mathrm{CPU}$, where orthogonal polynomials of up to fifth order are constructed numerically in each element with the error of recurrence coefficients being $\mathcal{O}\left(10^{-13}\right)$. Another choice is to make some standard meshes for commonly used random variables and organize the data into a table for future use. If the orthogonal chaos basis is available, we can implement gPC locally. For deterministic differential equations we have to guarantee the continuity (e.g., $C^{0}$ for $\nabla^{2}$ operators) on the interface between two adjacent elements in physical space. However, we do not have to worry about this for the stochastic case, because the probability measure of the points on the interface where $C^{0}$-type continuity in random space may not be satisfied is zero and the influence of such points on the interior values can be captured by the localized gPC. Furthermore, most statistics we are interested in, such as mean and variance, are defined as Lebesgue integrations, which implies that countable sets of zero measure have no contribution to the integration. This feature makes ME-gPC amenable to high-degree of parallelization and adaptivity.

\section{NUMERICAL EXAMPLES}

In this section we present some numerical examples of ME-gPC method including an algebraic equation and a typical ODE.

\subsection{A Simple Stochastic Algebraic Equation}

We reconsider the following stochastic algebraic equation given in [7]

$$
\hat{a}(\omega) u(\omega)=1,
$$

where $\hat{a}$ is a positive random variable in $[a, b]$ with an arbitrary probability measure. The reason we study this model is that it has many of the same essential properties as the following stochastic partial differential equation of elliptic type $[7,8]$

$$
\nabla \cdot(\hat{a}(\omega) \nabla u(\boldsymbol{x} ; \omega))=h(\boldsymbol{x}) .
$$

Let $\hat{a}$ be a general Beta random variable with PDF

$$
f(x)=\frac{(b-x)^{\alpha}(x-a)^{\beta}}{(b-a)^{\alpha+\beta+1} \hat{B}(\alpha+1, \beta+1)}, \quad a \leqslant x \leqslant b,
$$


where $\hat{B}\left(q_{1}, q_{2}\right)=\Gamma\left(q_{1}\right) \Gamma\left(q_{2}\right) / \Gamma\left(q_{1}+q_{2}\right)$ is the beta function.

Here we set $a=2, b=3, \alpha=0$ and $\beta=1$. To employ the standard Jacobi-chaos defined in $[-1,1]$ for $\hat{a}$, we first apply the following linear transform

$$
\hat{a}=\frac{1}{2} \xi+\frac{5}{2}
$$

Then we use Jacobi-chaos in terms of $\xi$ to solve Eq. (3.1). As for the ME-gPC, we construct numerically the orthogonal polynomial bases for elements $\left[a_{i}, b_{i}\right]$ of the decomposition of $[a, b]$ with respect to the corresponding conditional PDFs

$$
\hat{f_{i}}\left(x_{i}\right)=\frac{2\left(x_{i}-2\right)}{\left(b_{i}-a_{i}\right)\left(a_{i}+b_{i}-4\right)} .
$$

In Fig. 1, we compare ME-gPC with the Jacobi-chaos. It is well known that $\mathrm{gPC}$ relies on a three-dimensional table $\left\langle\Phi_{i} \Phi_{j} \Phi_{k}\right\rangle$ for Galerkin projection, where $\Phi_{i}$ is a basis mode of $\mathrm{gPC}$ and $\langle\cdot\rangle$ denotes the ensemble average. Here we set the integration error of table $\left\langle\Phi_{i} \Phi_{j} \Phi_{k}\right\rangle$ to be $10^{-14}$. It can be seen that the two error curves are perfectly matched

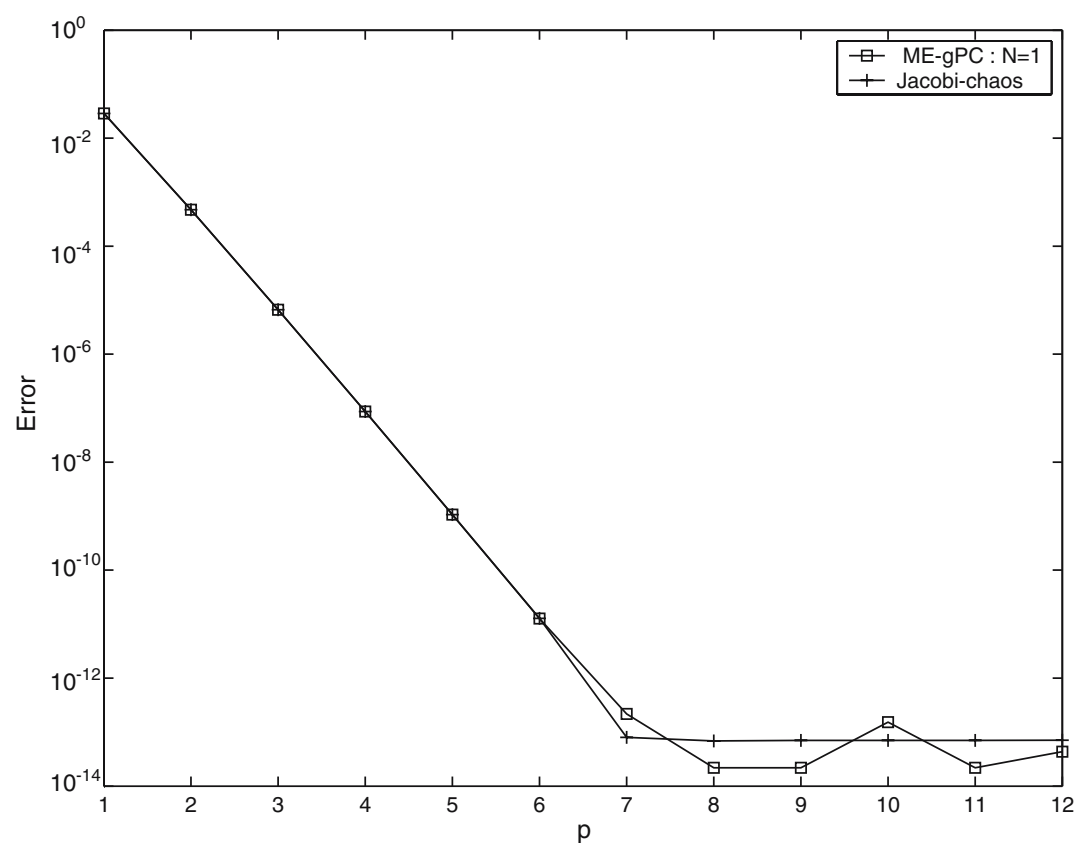

Fig. 1. Comparison of gPC and ME-gPC with one element. 
when $p$ is less than 6 . When the error is close to $10^{-14}$, the error curve of original gPC becomes flat while the error curve of ME-gPC has some fluctuation because the actual errors of the recurrence coefficients $\alpha_{i}$ and $\beta_{i}$ in Eq. (2.9) are a little bigger than $10^{-14}$. In Fig. 2, we show the $p$-convergence on the left and $h$-convergence on the right. For the $h$-convergence the index of algebraic convergence is about $2(p+1)$, which is consistent with the estimate given in [7]. In Fig. 3 the third- and fourthorder orthogonal polynomials and the corresponding (unscaled) PDFs of a two-element decomposition are shown. It can be seen that the polynomials with same order in different elements are slightly different.
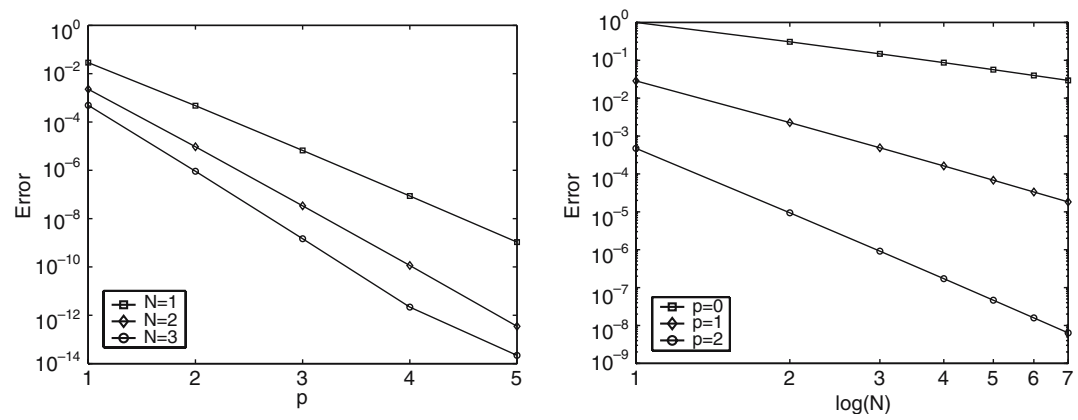

Fig. 2. Convergence of ME-gPC for the stochastic algebraic equation. Normalized errors of variance using uniform meshes. Left: $p$-convergence with $N$ being number of elements; Right: $h$-convergence with $p$ being polynomial order.
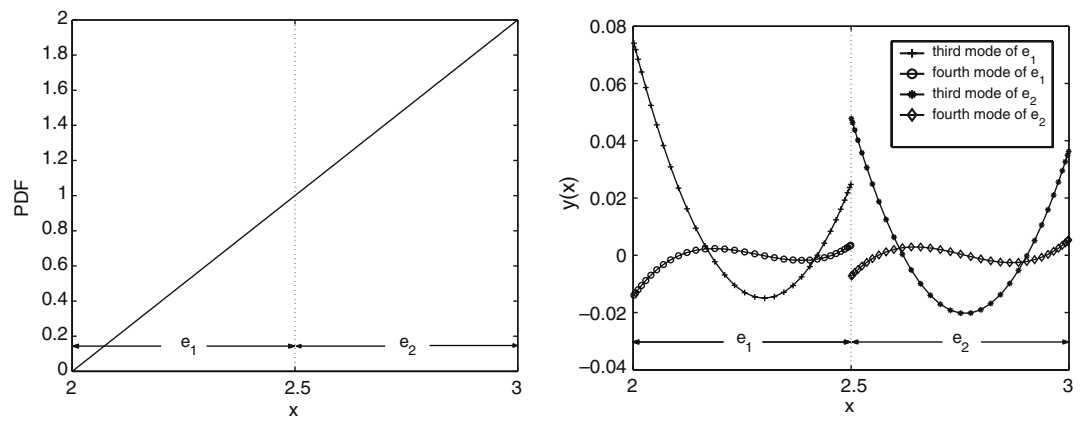

Fig. 3. A two-element decomposition for a Beta random variable on $[2,3]$ with $\alpha=0$ and $\beta=1$. Left: (unscaled) PDFs; Right: Third- and fourth-order orthogonal polynomials based on Gauss quadrature points. 


\subsection{One-Dimensional ODE Model}

Next we consider the following ODE equation studied with the original $\mathrm{gPC}$ in [4]

$$
\frac{\mathrm{d} u}{\mathrm{~d} t}=-\sigma \kappa(\omega) u, \quad u(0 ; \omega)=u_{0},
$$

where $\sigma$ and $u_{0}$ are constant and $\kappa(\omega)$ is a random variable of Beta distribution or Gaussian distribution. For the Beta distribution, the random variable is defined on $[-1,1]$. For the Gaussian distribution, we only use the middle element $[-6,6]$ and drop the tail elements since the contribution of the tail elements is neglectable in probability $\left(\operatorname{Pr}\left(I_{[6, \infty]}=1\right)=\right.$ $\left.9.87 \times 10^{-10}\right)$.

In Fig. 4 we show the $p$-convergence of ME-gPC for different random inputs. Comparing to the original gPC, the accuracy is significantly improved by increasing the number of random elements without losing the exponential convergence. In Fig. 5 we study the index of algebraic convergence ( $h$-convergence). The slope of straight solid lines is $-2 p-2$. We can see that the index of algebraic convergence of ME-gPC goes asymptotically to $2(p+1)$ as estimated. We show the third- and fourth-order orthogonal polynomials and the corresponding (unscaled) PDFs of a twoelement random decomposition in Fig. 6 for a Beta distribution $\mathrm{B} e(1,2)$.

\section{SUMMARY}

A multi-element generalized polynomial chaos (ME-gPC) method based on polynomial functionals is developed. Unlike any previous work,
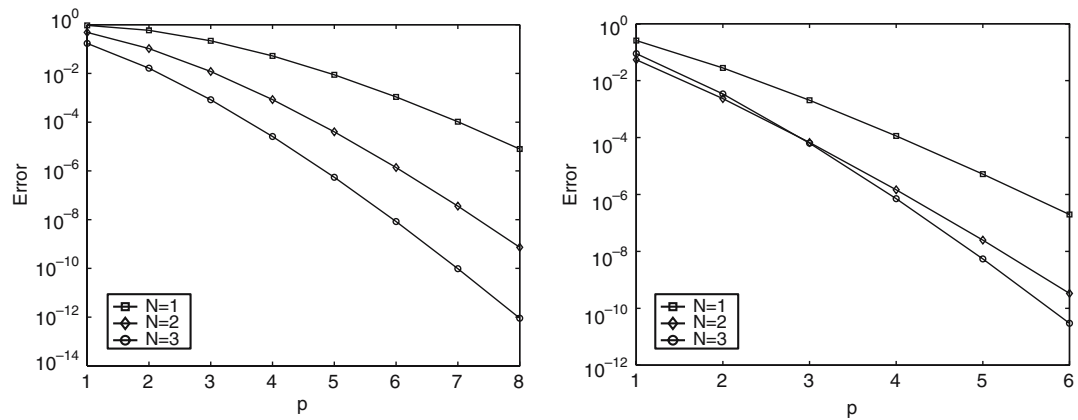

Fig. 4. $p$-convergence of ME-gPC for the ODE model. Normalized errors of variance using uniform meshes with $N$ random elements. Left: $\kappa$ is of $\operatorname{Beta}$ distribution $\operatorname{Be}(1,2)$ and $\sigma=1$; Right: $\kappa$ is of Gaussian distribution $\mathcal{N}(0,1)$ and $\sigma=0.1$. 

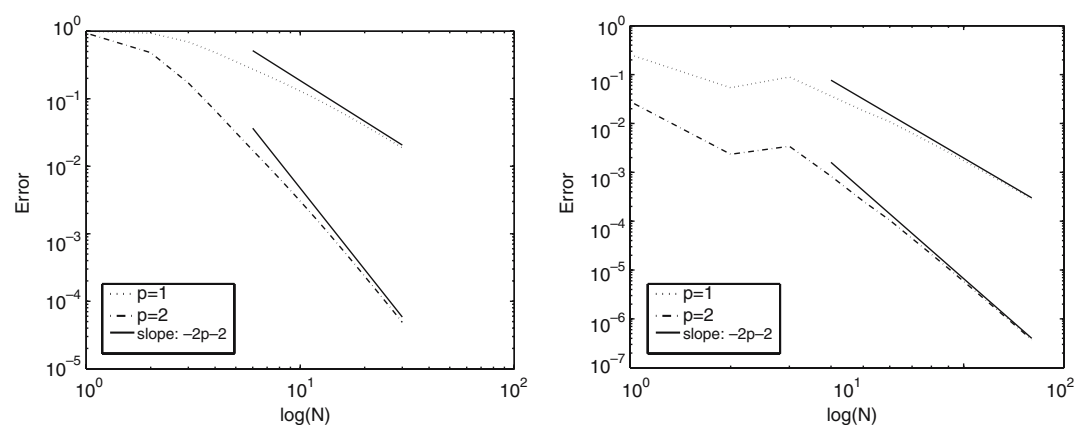

Fig. 5. Index of algebraic convergence of ME-gPC for the ODE model. Normalized errors of variance using uniform meshes. Left: $\kappa$ is of Beta distribution $\mathrm{Be}(1,2)$ and $\sigma=1$; Right: $\kappa$ is of Gaussian distribution $\mathcal{N}(0,1)$ and $\sigma=0.1$.
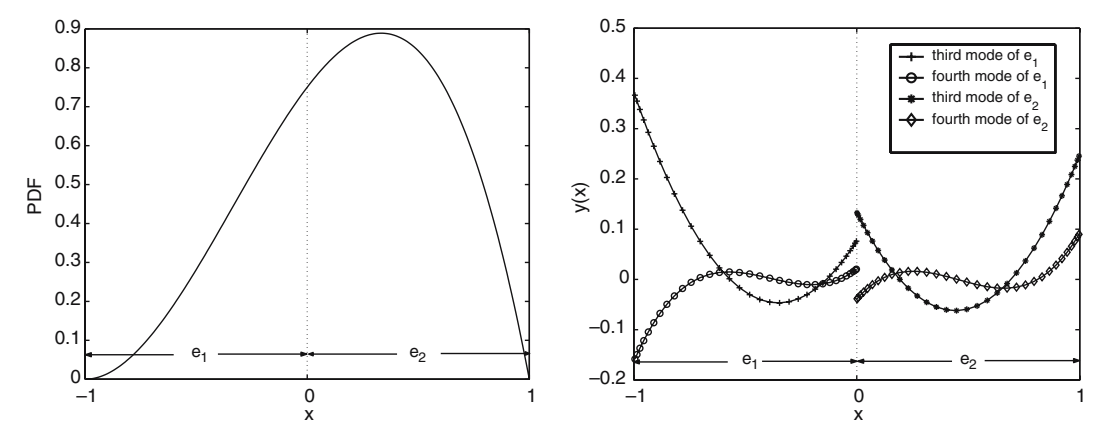

Fig. 6. A two-element decomposition for a Beta random variable on $[-1,1]$ with $\alpha=1$ and $\beta=2$. Left: (unscaled) PDFs; Right: Third- and fourth-order orthogonal polynomials based on Gauss quadrature points.

here we construct these polynomials on-the-fly based on the correspondence established in previous work between probability measures and orthogonality weights in the polynomial basis, [4, 15]. The ME-gPC method can achieve $h p$-convergence for any probability measure. In this first paper, we demonstrate the high accuracy and efficiency of the method using a simple algebraic equation and an ODE model, both of which have been used in previous work [7, 4]. Compared to the Monte Carlo method, the speedup of ME-gPC can be estimated from the $h p$-convergence $[9,7$, 4] because the local orthogonality can be maintained on-the-fly. For example, the $h$-type error $\mathcal{O}\left(N^{-2(p+1)}\right)$ of ME-gPC is much smaller than the error $\mathcal{O}\left(N^{-1 / 2}\right)$ of the Monte Carlo method. The current work provides the fundamental ideas for interfacing numerical simulation and experimental inputs with uncertainty, and thus the construction of new effective data 
assimilation techniques. It also provides the necessary approximation procedures for adaptive domain decomposition techniques in random space.

\section{ACKNOWLEDGEMENT}

This work was supported by AFOSR, DOE and NSF.

\section{REFERENCES}

1. Wiener, N. (1938). The homogeneous chaos. Amer. J. Math. 60, 897-936.

2. Ghanem, R. G., and Spanos, P. (1991). Stochastic Finite Eelements: A Spectral Approach, Springer-Verlag, New York.

3. Ghanem, R. G. (1999). Stochastic finite elements for heterogeneous media with multiple random non-gaussian properties. ASCE J. Eng. Mech. 125(1), 26-40.

4. Xiu, D., and Karniadakis, G. E. (2002). The Wiener-Askey polynomial chaos for stochastic differential equations. SIAM J. Sci. Comput. 24(2), 619-644.

5. Le Maitre, O. P., Njam, H. N., Ghanem, R. G., and Knio, O. M. (2004). Uncertainty propagation using Wiener-Haar expansions. J. Comput. Phys. 197, 28-57.

6. Le Maitre, O. P., Njam, H. N., Ghanem, R. G., and Knio, O. M. (2004). Multiresolution analysis of Wiener-type uncertainty propagation schemes. J. Comput. Phys. 197, 502-531.

7. Deb, M. K., Babuška, I., and Oden, J. T. (2001). Solution of stochastic partial differential equations using Galerkin finite element techniques. Comput. Methods Appl. Mech. Eng. 190, 6359-6372.

8. Babuška, I., and Chatzipantelidis, P. (2002). On solving elliptic stochastic partial differential equations. Comput. Methods Appl. Mech. Eng. 191, 4093-4122.

9. Babuška, I., Tempone, R., and Zouraris, G. E. (2004). Galerkin finite element approximations of stochastic elliptic differential equations. SIAM J. Numer. Anal. 42(2), 800825 .

10. Frauenfelder, P., Schwab, C., and Todor, R. A. Finite elements for elliptic problems with stochastic coefficients. Comput. Methods Appl. Mech. Eng. 194, 205-228.

11. Wan, X., and Karniadakis, G. E. (2005). An adaptive multi-element generalized polynomial chaos method for stochastic differential equations. J. Comput. Phys. 209(2), 617642.

12. Cameron, R. H., and Martin, W. T. (1947). The orthogonal development of nonlinear functionals in series of Fourier-Hermite functionals. Ann. Math. 48, 385.

13. Gautschi, W. (1982). On generating orthogonal polynomials. SIAM J. Sci. Stat. Comput. 3(3), 289-317.

14. Gautschi, W. (1994). Orthpol - a package of routines for generating orthogonal polynomials and gauss-type quadrature rules. ACM Trans. Math. Softw. 20(1), 21-62.

15. Schoutens, W. (1999). Stochastic Processes in the Askey Scheme. PhD thesis, K.U. Leuven. 